\title{
Academic Detailing From Medical Students: Part of a Plan to Emphasize Feedback in One Clerkship
}

Robert P. Wilfahrt, MD | Sara S. Oberhelman, MD | Zachary T. Merten, MD | Kurt B. Angstman, MD

PRIMER. 2019;3:26.

Published: 11/20/2019 | DOI: 10.22454/PRiMER.2019.469608

\section{Abstract}

Introduction: Medical schools have an enduring need to provide ongoing faculty development and to foster educational alliances between teachers and learners, so that feedback provided to learners is both frequent and of high quality. We hypothesized that medical students trained as academic detailers with a mission to increase the emphasis on feedback could serve in this role during our clerkship, while still being evaluated as students in our clerkship rotation.

Methods: The family medicine clerkship at Mayo Clinic School of Medicine launched a revised curriculum in 2016 in which students were taught how they might build an educational alliance with preceptors, were taught characteristics of high-quality feedback, and practiced requesting more useful feedback when initial quality was poor. After utilizing a clerkship-specific curriculum with small group sessions on receiving feedback, and training students and preceptors on the SNAPPs model, students were then directed to request feedback from their preceptors and model successful feedback conversations for preceptors. The study evaluated the medical students' summative evaluations to compare the rate from the preintervention year (2015-2016) and the intervention year (2016-2017) at which preceptors added comments on students' use of feedback.

Results: Preceptors' written comments about students seeking feedback increased at about a four-fold rate $(74.4 \%$ vs $18.8 \%, P<.001)$ after implementing a suite of changes to our clerkship curriculum.

Conclusions: Using medical students to change preceptor behaviors was an important part of our suite of interventions. This intervention directed preceptor attention toward our instructional goal of increased medical student feedback.

\section{Introduction}

Feedback-an intentional conversation about a learner's performance used as a basis for improvement-is recognized as fundamental for the continued development of health care students. ${ }^{1}$ Academic detailing is the practice of delivering developmental material to community faculty using methods mimicking those of pharmaceutical salespeople. ${ }^{2}$ Academic detailing has been demonstrated to be favorable for preceptor training compared to centralized meetings occurring away from the clinician's usual practice..$^{3,4}$

Academic detailing has been practiced in Europe since the 1980s, but was first demonstrated successfully in the United States by Moser et al in 2004 for their Wichita-based family medicine faculty development project. ${ }^{5}$ The project's trainer typically provided lunch or snacks and began conversations with rapport-building of the preceptor faculty. This was followed by an assessment of the preceptor's individual needs. The project trainer could then select a focused educational intervention. Finally, the project trainer gave the community preceptor a thank-you trinket. This model utilized preceptor time effectively, but with more interpersonal interactions and a dollar cost that 
compared favorably to group workshops.

Academic detailing has also been utilized by giving medical students specific content training before they met community preceptors to deliver the content. ${ }^{6}$ However, this contact occurred outside of the usual academic schedule, while the students were not a part of the preceptors' current clerkships. Others have developed general guidelines for students as they enter clerkships. ${ }^{7}$

Based on reviews describing students' appreciation of the importance of feedback ${ }^{8}$ and their ability to identify characteristics of well-delivered feedback, ${ }^{9}$ we postulated that students would be motivated to obtain high-quality feedback and that students could serve as academic detailers to their own family medicine preceptor, while being taught by them. Our prior experience with faculty development suggested that student behaviors were more likely to be changed, and that by emphasizing this half of the feedback dyad we would reap greater changes in communication. We hypothesized an increase in the attention given to feedback via students' academic detailing during our clerkship would increase the frequency of feedback given by the preceptors.

\section{Methods}

In July 2016, the family medicine department at the Mayo Clinic Alix School of Medicine changed the curriculum (Table 1) to improve feedback provision and usage in a redesign of its third-year clerkship. Each medical school class had approximately 50 students who rotated through this 3-week clerkship, in 8 to 10 blocs during the academic year. We assigned the medical students to one or two preceptors at sites within Southeastern Minnesota, Jacksonville, Florida, or Phoenix, Arizona. The number of preceptors participating in this clerkship did not significantly change in the observation period, with a majority (56/62) participating in both years.

For the intervention, we began a clerkship curricular element with small group sessions including illustrative scenarios and videos. Students gave examples of good and bad feedback they had received, and characteristics of excellent feedback were listed. Barriers to successfully receiving feedback were discussed, as were means of improving the utility of awkwardly-offered feedback. The students were then directed to request feedback from their preceptors and to model successful feedback conversations for preceptors.

The students also received training on the SNAPPS model of student presentation. ${ }^{10}$ SNAPPS differs from the traditional format of patient presentation usually taught in medical school. It reduces a student's usual emphasis on a detailed recounting of the history of present illness and instead makes a place for communication of the student's narrowed differential, analysis, and plan in order to help demonstrate higher-order thinking. The SNAPPS model was demonstrated to preceptors during departmental educational conferences and by online videos. Preceptors were reminded to employ the model at the beginning of each rotation. Faculty in-services were used to teach how to provide high-quality written assessments of students, and to alert preceptors of upcoming changes in the clerkship including use of students as academic detailers. However, we did not track which preceptors were present or which methods they used (conference, online, email, etc). We considered the students to be the more impacted audience, since their education was directly affected.

We altered clerkship grading criteria to include the preceptors' judgement on students' observed use of feedback. Within 4 weeks of the rotation's end, preceptors were required to describe student progress in a content-rich, freetext narrative assessment. The narrative content of these evaluations were then reviewed by one of the authors (R.P.W.) for any mention of words or phrases such as "sought feedback," or "took instruction well." The number of written narratives mentioning feedback was then tallied.

We considered all final course evaluations for the family medicine clerkship for the 2015-2016 (preintervention, $\mathrm{n}=49$ ) and 2016-2017 (intervention, $\mathrm{n}=47$ ) academic years. Our institutional review board considered this study exempt. We analyzed data by $\chi^{2}$ analysis with significance set at $P<.05$.

\section{Results}

In 2015-2016 there were nine assessments of seeking feedback for the 49 students (18.4\%). There was a significant 
increase to 35 experiences of feedback being mentioned in the narrative comments $(74.4 \%, P<.001)$ after the intervention in 2016-2017. Table 2 documents several representative comments from the faculty after the intervention.

\title{
Discussion
}

Our feedback-focused clerkship changes sought to meet entrustability targets for professional communication. Using a suite of tools to inform the medical student about the importance of feedback and train them in academic detailing, our study demonstrated a significant short-term increase in medical student feedback that was timely and formative.

The increased number of preceptor evaluations mentioning feedback shows that our preceptors noticed when students asked for feedback. As academic detailers, our students were more likely to develop an educational alliance with their preceptor. This alliance, together with prior exposure to tools allowing student-detailers to use feedback effectively, suggests that our feedback curriculum can improve students' educational experiences and ongoing performance. Using the students themselves to change preceptor behaviors was an important part of our suite of interventions-a suite that has successfully directed preceptor attention toward the instructional goal. The success of the intervention is demonstrated by the increase in the number of times it is mentioned by preceptors in their summative narration. The etiology of the improvement (whether increased student responsiveness or preceptor activation) is not the primary concern, but the result of enhanced timely clerkship feedback is.

\section{Tables and Figures}

Table 1: Components of Family Medicine Feedback Curriculum

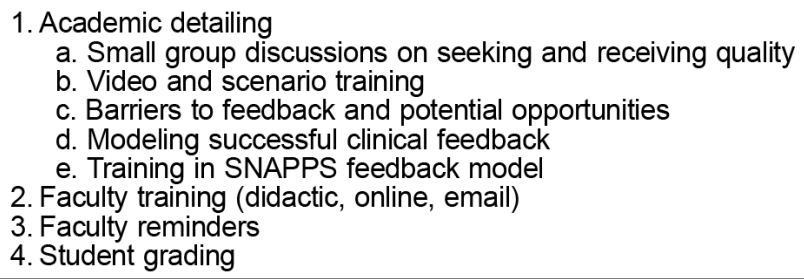

Table 2: Samples of Preceptors' Narrative Summative Evaluations

"... I appreciated that [student] would frequently ask for feedback, and he was receptive to constructive criticism... His presentation skills also improved throughout the rotation. Initially he had minor difficulties with obtaining all the information, but by the end of the rotation, he was able to provide very detailed histories."

"...[student] is gracefully accepting of feedback... which she then clearly attempted to incorporate."

"She engaged with feedback at multiple points during the rotation, sought to clarify feedback when needed, and was noted... to have clearly applied feedback to subsequent patient interactions."

\section{Corresponding Author}

\author{
Kurt B. Angstman, MD
}

Department of Family Medicine, Mayo Clinic, 2001 st St SW, Rochester, MN 55905. 507-284-2500. Fax:

507-266-4733.

angstman.kurt@mayo.edu

\section{Author Affiliations}

Robert P. Wilfahrt, MD - Department of Family Medicine, Mayo Clinic, Rochester, MN 
Sara S. Oberhelman, MD - Department of Family Medicine, Mayo Clinic, Rochester, MN

Zachary T. Merten, MD - Department of Family Medicine, Mayo Clinic, Eau Claire, WI

Kurt B. Angstman, MD - Department of Family Medicine, Mayo Clinic, Rochester, MN

\section{References}

1. Cantillon P, Sargeant J. Giving feedback in clinical settings. BMJ. 2008;337(nov10 2):a1961. https://doi.org /10.1136/bmj.a1961

2. Soumerai SB, Avorn J. Principles of educational outreach ('academic detailing') to improve clinical decision making. JAMA. 1990;263(4):549-556. https://doi.org/10.1001/jama.1990.03440040088034

3. Figueiras A, Sastre I, Tato F, et al. One-to-one versus group sessions to improve prescription in primary care: a pragmatic randomized controlled trial. Med Care. 2001;39(2):158-167. https://doi.org/10.1097 /00005650-200102000-00006

4. Habraken H, Janssens I, Soenen K, van Driel M, Lannoy J, Bogaert M. Pilot study on the feasibility and acceptability of academic detailing in general practice. Eur J Clin Pharmacol. 2003;59(3):253-260. https://doi.org/10.1007/s00228-003-0602-6

5. Moser SE, Dorsch JN, Kellerman R. The RAFT approach to academic detailing with preceptors. Fam Med. 2004;36(5):316-318.

6. Blitz DA, Mallen JR, Kwiatkowski TG, Rabin JM, Dlugacz YD, Silverman RA. Not for industry only: medical students and office-based academic detailing the PIVOT (Pregnant women Influenza Vaccine Optimization Team) initiative. Adv Med Educ Pract. 2015;6:323-327. https://doi.org/10.2147/AMEP.S72384

7. Hoffman $M$, Cohen-Osher $M$. The one-minute learner: evaluation of a new tool to promote discussion of medical student goals and expectations in clinical learning environments. Fam Med. 2016;48(3):222-225.

8. Clynes MP, Raftery SE. Feedback: an essential element of student learning in clinical practice. Nurse Educ Pract. 2008;8(6):405-411. https://doi.org/10.1016/j.nepr.2008.02.003

9. Cahill HA. A qualitative analysis of student nurses' experiences of mentorship. J Adv Nurs. 1996;24(4):791-799. https://doi.org/10.1046/j.1365-2648.1996.25618.x

10. Wolpaw TM, Wolpaw DR, Papp KK. SNAPPS: a learner-centered model for outpatient education. Acad Med. 2003;78(9):893-898. https://doi.org/10.1097/00001888-200309000-00010

Copyright $@ 2019$ by the Society of Teachers of Family Medicine 